\title{
Phase diagram of a binary mixture in a closed cavity
}

\author{
A. Vorobev ${ }^{1}$, D. Lyubimov ${ }^{2}$, T. Lyubimova ${ }^{2,3}$ \\ ${ }^{1}$ Energy and Technology Research Group, Faculty of Engineering Sciences and the Environment, \\ University of Southampton, Southampton SO17 1BJ, United Kingdom \\ ${ }^{2}$ Perm State University, Perm 614600, Russia and \\ ${ }^{3}$ Institute of Continuous Media Mechanics, Perm 614013, Russia
}

(Dated: December 16, 2016)

\begin{abstract}
Normally, the phase diagram is reported as property of the binary mixture. We show that the phase diagram (that is the zones of thermodynamic stability of the states of the binary mixture) is also affected by the size of the container. We investigate the thermodynamic stability of the binary mixture in a closed cavity, and identify the zone in parameters where the binary mixture is heterogeneous in equilibrium (the zone of spinodal decomposition), the zone where the mixture is always homogeneous in equilibrium, and the zone where the transition between these two state is possible (the metastable nucleation zone). In addition, we investigate the properties of the minimum single droplet that may be in equilibrium in the closed cavity (for the given average concentration, all smaller droplets would always dissolve). We show that the size of such droplets depends on the cavity's size, as $\sim L^{1 / 2}$.
\end{abstract}

\section{INTRODUCTION}

Currently, there is an interest in understanding of physical processes taking place in micro-enclosures (with typical sizes of micrometers), e.g. mixing/de-mixing in micro-reactors used for new bioengineering applications [1], or e.g. cryo-preservation when formation/melting of icicles in tissue cells occur [2,3]. Small dimensions of the enclosures may affect both the stability of the phase states of the mixture and the rate of growth/decay of nuclei.

Sizes of the nuclei are presumed to be negligibly small in comparison with the container's dimensions, so, in particular, the changes in concentration of a surrounding medium upon nucleation are usually disregarded. This assumption was first made by Gibbs [4] and also accepted by other researchers [5]. For the micro-enclosures this condition may however become invalid. In addition, it is also known that the size of a critical nucleus depends on thermodynamic conditions, in particular on temperature, and e.g. the size of a nucleus formed in a near critical medium may be considerably larger, comparable to the size of the cavity.

In the classical theory, a nucleus of the new phase is thermodynamically unstable, which can be invalid in the limit of very small nuclei. The surface tension coefficient of sufficiently small droplets should depend on the droplet's volume, at least in order to exclude the unbounded growth of the inner droplet's pressure. The necessity for such dependence was first suggested by Gibbs [4], and later Tolman [6] proposed the following simple formula,

$$
\sigma=\frac{\sigma_{\infty}}{1+2 \delta_{T} / R_{0}}
$$

where $\delta_{T}$ is a phenomenological parameter called Tolman's length, which value was estimated by Tolman as
$10^{-6} \mathrm{~m}$. Numerous other formulae were proposed later [7-11]. The phase-field description of the multiphase medium incorporates the dependence of the surface tension on the droplet's size. In [12], on the basis of the phase-field approach, it was shown that sufficiently small droplets may actually be stable.

Additionally, the classical conclusion on the instability of nuclei may also change for a binary mixture in a closed container. A smaller nucleus formed in a closed container would be unstable as it does not fill the influence of the walls. Its growth however is accompanied by depletion of the solute from the solvent-filled phase and its final size would be bounded by the total amount of the solute available in the container. A droplet becomes stable when it reaches the critical size. This has been studied in [13] on the basis of the Laplace approach and with the use of the Van der Waals equation of state. It was found that the thermodynamic and hydrodynamic stability of the droplet is defined by two parameters, average density, and surface tension. If the average density is low then the system is homogeneous in equilibrium. At some critical average density there appear two solutions: one solution corresponds to a smaller unstable droplet, while a bigger droplet may be stable. Upon further growth of the average density only one (bigger droplet) solution remains.

In the current work, the thermodynamic stability of the binary mixture enclosed in a finite-size container is studied. We also investigate the properties of an equilibrium nucleus. The problem is examined on the basis of the phase-field approach that is used to represent the different states of the binary mixture. Such an approach is universal, frequently used for modelling the systems with phase transitions of different nature, and hence is capable of producing the results applicable for description of e.g. the evolution of liquid nuclei in vapour or the formation/melting of icicles, etc. 
We should also mention that there are a large number of papers that examine the problem of nucleation in confined space (e.g. [14-18]). The different alternative approaches have been used for these studies: the molecular dynamics and Monte Carlo simulations [14], the density functional theory [15], the solution of kinetic equations [17], and the generalised Gibbs' approach [16, 18]. The primary focus of all these papers is though on the work needed for formation of the critical cluster and on the nucleation rates, i.e. primarily on the shape and development of the precursor clusters that lead the phase separation. The focus of our work is different. We primarily aim to identify the conditions when the nucleation is at all possible.

\section{PROBLEM STATEMENT}

Within the phase-field approach a phase boundary is represented as a transitional layer across which physical properties change sharply but still continuously. To derive equations for such a medium the internal energy is assumed to be a function of concentration, and also of concentration gradient. This was first done by Van der Waals [19],

$$
f(C, \nabla C)=f_{0}(C)+\frac{\epsilon}{2}(\nabla C)^{2},
$$

where $f$ is the specific free energy function of the mixture with $f_{0}$ being its classical part, and $\epsilon$ is the capillary coefficient.

The full governing equations that define the thermoand hydrodynamic evolution of an isothermal binary mixture were first derived by Lowengrub and Truskinovskiy [20]. These are the so-called Cahn-Hilliard-Navier-Stokes equations. The equations include the balances of mass, linear momentum, and species. Owing to dependence of the mixture density on concentration, the full continuity equation is required to describe the evolution of binary mixture of two incompressible liquids, and gradients of chemical potential can induce non-solenoidal velocity field. By filtering out these quasi-acoustic effects, the full equations can be simplified for consideration of slower diffusive and convective mass transports [21].

Our current analysis is restricted to the thermodynamic evolution of an isothermal binary mixture defined by the single diffusion equation,

$$
\frac{\partial C}{\partial t}=\Delta \mu
$$

Here $\mu$ is the chemical potential that is defined as

$$
\mu=\mu_{0}-\epsilon \Delta C, \quad \mu_{0} \equiv \frac{d f_{0}}{d C} .
$$

The gravity effects, which include the barodiffusion flux [21], are disregarded in the current work.
For the classical part of the free energy function, a 'double well potential' that permits modelling two-phase states is used,

$$
f_{0}=a\left(C-C_{c}\right)^{2}+b\left(C-C_{c}\right)^{4}
$$

This approximation was introduced for description of a near-critical system [22]. Here $a$ and $b$ are the two phenomenological parameters. It can be shown that for a near-critical system, $a \sim\left(T-T_{c}\right)$, i.e. this parameter can be either positive or negative depending whether the system's temperature is above or below the critical temperature, while $b$ is always positive. We however use this free energy function as a model for a binary mixture that may experience a phase transition (being either heterogeneous or homogeneous), and we do not obligatory assume that a mixture temperature is close to the critical point.

For further analysis it is convenient to change the definition of concentration by shifting, $C \rightarrow\left(C-C_{c}\right)$. The equations are also non-dimensionalized by using the size of a container, $L$, as a length unit and the diffusive time scale, $L^{2} / \mu_{*}$, as a time unit, where $\mu_{*}=b$ is a unit of the chemical potential.

Finally, the governing equation to be solved reads as follows

$$
\begin{array}{r}
\frac{\partial C}{\partial t}=\Delta \mu, \\
\mu=\mu_{0}-\mathrm{Ca} \Delta C=2 A C+4 C^{3}-\mathrm{Ca} \Delta C,
\end{array}
$$

where $\mu_{0} \equiv d f_{0} / d C$. Here we also introduce two nondimensional parameters. $A \equiv a / b$ defines the thermodynamic model for a binary mixture. It can be shown that for a flat interface separating two semi-infinite liquid domains, the minima of the free energy functions are defined by $\pm\left(-\frac{A}{2}\right)^{1 / 2}$. In the current work, we assume that $A=-\frac{1}{2}$, which gives $\pm \frac{1}{2}$ for the minima of the free energy function, and these two values of concentration would be associated with the two pure components of the mixture. The second non-dimensional parameter is the capillary parameter, $\mathrm{Ca}=\epsilon /\left(L^{2} b\right)$, that defines the role of capillary effects.

Equation (6) is supplemented with the following boundary conditions,

$$
\frac{\partial \mu}{\partial n}=0, \quad \frac{\partial C}{\partial n}=0
$$

first of which signifies no diffusive flux through container's walls, while the second condition defines the wetting conditions on the wall, namely, it states that the molecules of the wall are neutral to the molecules of mixture components [21]. Here $\vec{n}$ is the unit vector orthogonal to the wall, and $\partial / \partial n$ is the normal derivative. 


\section{RESULTS}

\section{Linear stability theory}

The mathematical problem stated above has both homogeneous and non-homogeneous solutions. The homogeneous solutions are trivial, with uniform concentration field, $C=q$, where $q$ is the average concentration of the mixture, $q \equiv \frac{1}{V} \int_{V} C d V$ ( $V$ is the total volume of the container).

Let us first investigate the linear stability of the homogeneous solution. For simplicity, we restrict the linear stability analysis to a 1D problem, assuming that a mixture fills a spherical container, and the evolution of a spherical nucleus of the new phase positioned in the centre of the container is studied. For such a geometry, the linear stability problem is defined by the equation,

$$
\frac{\partial C^{\prime}}{\partial t}=\frac{1}{r^{2}} \frac{\partial}{\partial r}\left[r^{2} \frac{\partial}{\partial r}\left(D_{0} C^{\prime}-\mathrm{Ca} \frac{1}{r^{2}} \frac{\partial}{\partial r}\left(r^{2} \frac{\partial C^{\prime}}{\partial r}\right)\right)\right] .
$$

Here, $C^{\prime}$ stands for the small perturbation of a uniform state, $C \rightarrow q+C^{\prime}$, and $D_{0} \equiv 2 A+12 q^{2}$ is the diffusion coefficient.

The boundary conditions are the boundedness of the solution at the centre $(r=0)$ and the conditions (8) at the container's wall, namely $\frac{\partial \mu}{\partial r}=0$ and $\frac{\partial C}{\partial r}=0$ at $r=1$.

The linear stability in respect to the perturbations of the following form

$$
C^{\prime} \sim \frac{\sin (p r)}{r} \exp (\lambda t)
$$

is examined. Such perturbations satisfy the boundary conditions, if

$$
p \cos (p)=\sin (p)
$$

The smallest non-trivial solution of equation (11) is $p_{0}=$ 4.49 , which represents a perturbation in the form of a single droplet positioned in the centre of the container.

The homogeneous solution becomes unstable in respect to the perturbations (10) when

$$
|q|<\sqrt{\frac{1}{12}\left(-2 A-\mathrm{Ca} p_{0}^{2}\right)} .
$$

Obviously, a non-homogeneous solution can be realized if $\mathrm{Ca} \leq(-2 A) / p_{0}^{2}$, if however $\mathrm{Ca} \leq(-2 A) / p_{1}^{2}$, with $p_{1}=7.73$ then three-layer structures (e.g. solute-solventsolute) can be also realized. For containers of bigger dimensions, even more complex structures are possible. Though, all these multi-layered structures are obviously meta-stable, and would correspond to higher values of the total free energy.[27]

For a plane geometry, i.e. a plane interface separating two liquids enclosed in a plane layer, the stability diagram would be the same equation (12) with $p_{0}=\pi$.
Formula (12) defines the boundary of the spinodal decomposition when the homogeneous state is unstable to any infinitely small perturbation. This boundary is shown in figure 1a with a dashed line.

\section{Numerical results}

The full non-linear stability of the homogeneous state was studied for the binary mixture that fills a $2 \mathrm{D}$ square cavity. Equation (6) was solved numerically using the finite-difference approach and a uniform grid. The runs with different resolutions were fulfilled to verify the gridindependence of the obtained results. The equilibrium interface thickness is proportional to $\sqrt{-\mathrm{Ca} / A}$. All further results are obtained for $A=-1 / 2$, and the grid resolution was adjusted to the values of the capillary number.

We investigate the stability of the homogeneous solution in respect to a droplet-like perturbation. Namely, all numerical runs were initiated by the following concentration field

$$
C_{0}(x, y)=\beta \tanh \left(\frac{r-r_{0}}{\delta_{0}}\right)
$$

which represents a spherical droplet of an initial radius $r_{0}$ positioned in the centre of the cavity. Here, $x$ and $y$ are the two Cartesian coordinates, $r$ is the distance from the cavity's centre, and $\delta_{0}$ is the initial interface thickness (that was approximately set equal to $\sqrt{-C a / A}$, which corresponds to the thickness of a thermodynamically equilibrium flat interface). The amplitude of perturbation, defined by $\beta$, is adjusted so to provide the needed average concentration of the mixture in the container, $q=\frac{1}{V} \int_{V} C_{0} d V$.

Profile (13) is only an approximation to the solution of the governing equation (6). During the evolution, the droplet set by expression (13) either disappears, which means that the equilibrium state is stable in respect to such a perturbation, or droplet (13) evolves to a new equilibrium droplet with radius and interface thickness slightly different from the initial values. The changes between the initial and final droplets are stronger for larger capillary numbers. For lower capillary numbers, the concentration profile (13) was found to be a rather good approximation to the exact solution of equation (6).

To characterise the evolution of the multiphase mixture, the number of parameters was used, these are the droplet's radius $r_{d}$, the surface tension $\sigma$ and the interface thickness $\delta,[28]$

$$
\begin{array}{r}
r_{d} \equiv \frac{\int_{V} r(\nabla C)^{2} d V}{\int_{V}(\nabla C)^{2} d V}, \\
\sigma \equiv \frac{C a}{2 \pi r_{d}} \int_{V}(\nabla C)^{2} d V,
\end{array}
$$




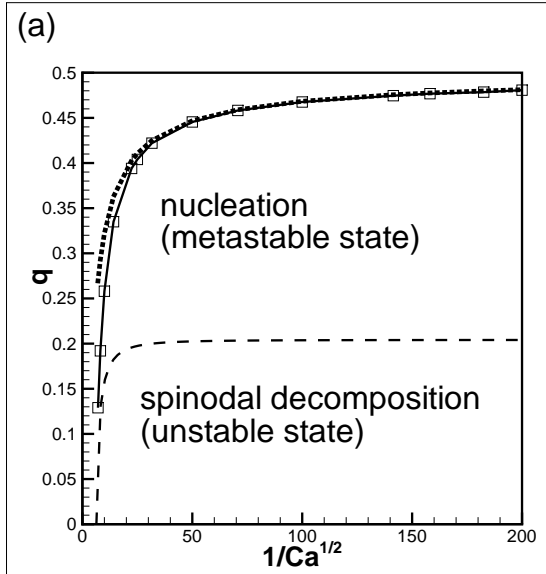

(d)

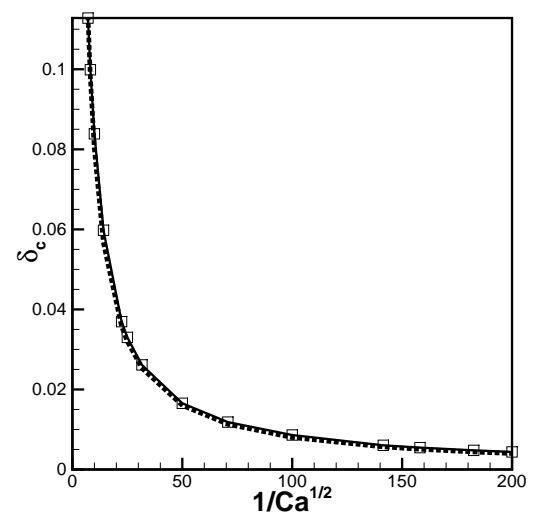

(b)

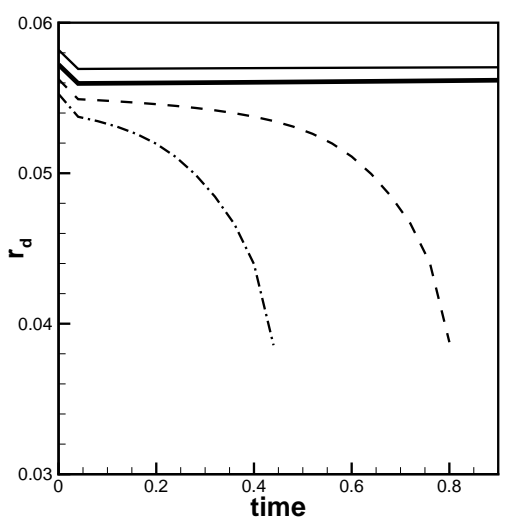

(e)

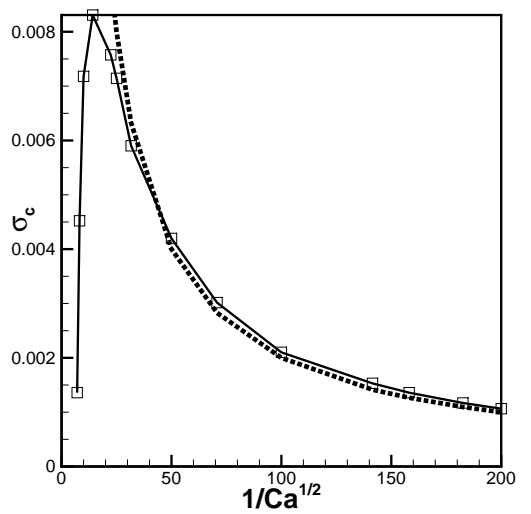

(c)

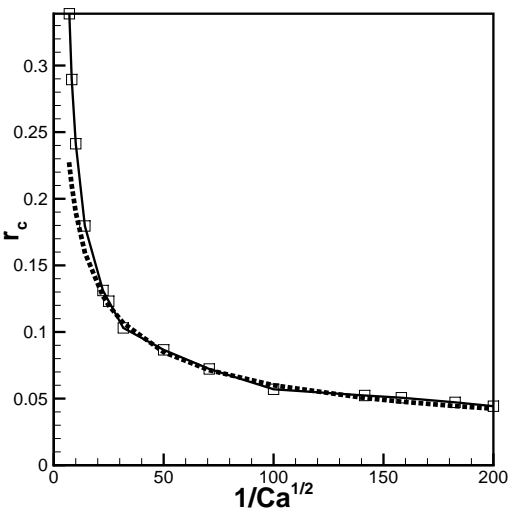

(f)

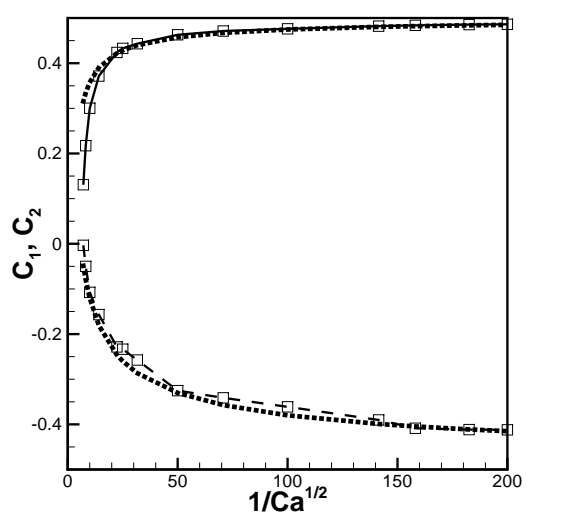

FIG. 1: (a) The phase diagram for a binary mixture in a closed cavity. The dashed line depicts the boundary of the zone of spinodal instability (a homogeneous state is unstable to infinitely small perturbations), the solid line defines the zone of nucleation (a homogeneous state is stable to infinitely small perturbations but unstable to finite-size perturbations). The homogeneous state is absolutely stable above the solid line. The dotted line is $0.5-\mathrm{Ca}^{3 / 8}$. (b) The time dependences of the radii of different droplet-like perturbations. The thick line corresponds to the evolution of the droplet of the critical size $\left(r_{0}=r_{c}=0.057\right)$. The thin solid curve corresponds to the droplet with radius slightly bigger than the critical radius $\left(r_{0}=0.058\right)$, and the thin dashed and dash-dot lines correspond to the droplets with radii slightly smaller than the critical one $\left(r_{0}=0.056\right.$ and $\left.r_{0}=0.055\right)$. These curves are obtained for $q=0.4676$ and $C a=10^{-4}$. (c,d,e) The size, interface thickness and surface tension of the critical droplet (the smallest single droplet that can remain in equilibrium in a closed container). The dotted lines in (c) depict the curve $0.6 \mathrm{Ca}^{1 / 4}$, in (d) $0.8 \mathrm{Ca}^{1 / 2}$ and in (e) $0.2 \mathrm{Ca}^{1 / 2}$. (f) The average concentrations in the droplet phase (dashed line) and outside (solid line) the droplet. The result is obtained for the critical droplets. The dotted lines depict the curves, $-0.5+1.2 \mathrm{Ca}^{1 / 4}$ and $0.5-0.8 \mathrm{Ca}^{3 / 8}$.

$$
\delta^{2} \equiv \frac{\int_{V}\left(r-r_{d}\right)^{2}(\nabla C)^{2} d V}{\int_{V}(\nabla C)^{2} d V}
$$

The phase diagram can be set by two parameters, the average concentration of the mixture enclosed by the cavity and the capillary number.

Firstly, through the set of numerical runs we found that if the average concentration is closer to zero (solute and solvent are introduced into the container in approximately equal amounts), then the homogeneous state is unconditionally unstable (for the accepted value of the parameter $A$ ), and the mixture separates into two phases following the spinodal decomposition. In the case of the spinodal instability, multiple smaller nuclei are initially formed within the cavity, the later growth of some of these nuclei leads to an equilibrium two-phase state of the mixture. This process of spontaneous decomposition has been already studied on the basis of the phase-field approach (see e.g. [23]), and we report no further details on the dynamics of this process. What is important for us, that the binary mixture is always heterogeneous in equilibrium if its parameters correspond to the zone of the spinodal decomposition.

Secondly, we found that the zone of instability of the homogeneous state is in fact much wider, if the stability in respect to finite-size perturbations is examined. The solid line in figure 1a depicts the nucleation curve that separates the zone of instability of the homogeneous state 
in respect to droplet-like nuclei of finite size ('the critical cluster' for the classical nucleation theory). The size of the minimum perturbation capable of turning the homogeneous state into a heterogeneous one depends on the average concentration and capillary number, growing if the average concentration, $q$, is increased. The maximum value of the minimum perturbation is on the nucleation curve. For the parameters that correspond to a point on the nucleation curve, we show that smaller droplets dissolve, but the dissolution rate of a droplet depends on its size, so droplets with the sizes closer the critical one dissolves slower, as can be seen figure 1b. The droplet of the critical size remains thermodynamically stable, and this applies for all droplets with the sizes bigger than the critical one. Thus, the critical droplet with the parameters on the nucleation curve would also represent the smallest single droplet that may remain in the cavity in equilibrium.

Figures 1c,d,e depict the characteristics of the critical droplets (for the droplets with the parameters on the nucleation curve). The dependence of the size of such droplets on the capillary number is shown in figure 1c. For larger capillary numbers the critical radius is quite large, and for very large capillary numbers the walls of the container would even affect the droplet's shape. If however the capillary number is smaller, the size of the droplet decreases. We found that the critical size (for the parameters on the nucleation curve) depends on the capillary number as $r_{c} \approx 0.6 \mathrm{Ca}^{1 / 4}$. As estimated in [24], the typical value of the capillary number for a miscible system should be of the order of $\mathrm{Ca} \sim 10^{-5}$, which indicates that the smallest droplet that may remain in equilibrium for a typical system is below 0.03 (in the units of the container's size). Dependences of the surface tension and thickness of the phase boundary against the capillary number are shown in Figure 1d,e. For smaller capillary numbers, the interface becomes thinner, and the interfacial tension decreases, following the dependences, $\delta_{c} \sim 0.8 \sqrt{\mathrm{Ca}}$ and $\sigma_{c} \sim 0.2 \sqrt{\mathrm{Ca}}$. The decrease of the surface tension at very large capillary numbers should likely be explained by the influence of the walls on the droplet shape.

Finally, we also show the average concentrations in the equilibrium droplets of the critical size, inside the droplet, $C_{1}$, and in the medium outside the droplet, $C_{2}$. Since the droplets are generally small, the average concentration in the ambient medium is closer to $1 / 2$ (which is the value of the pure component). Inside the droplet though, the value of the average concentration is different from the value that would correspond to a pure component, $-1 / 2$. The obtained curves could be asymptotically represented by the following dependences, $C_{1}=-0.5+1.2 \mathrm{Ca}^{1 / 4}$ and $C_{2}=0.5-0.8 \mathrm{Ca}^{3 / 8}$. The differences in the concentrations $C_{1}$ and $C_{2}$ from the values $\pm 1 / 2$ should be explained by the capillary effects.

\section{CONCLUSIONS}

On the basis of the phase-field approach we have examined the phase stability of the liquid/liquid binary mixture that fills a closed cavity. The mixture can be either homogeneous (the liquids fully mix) or heterogeneous (there exists an interface separating the liquids). The stability of these two states of the mixture is defined by the average concentration $q$ and by the capillary number Ca. The resultant phase diagram is depicted in figure 1a, which includes the boundaries of the spinodal decomposition (when the homogeneous state is absolutely unstable) and the nucleation line (when the homogeneous state is unstable in respect to finite-size droplet-like perturbations). If the average concentration of the mixture is in the range of the nucleation zone, then the binary mixture is metastable, i.e. can be either homogeneous or heterogeneous in equilibrium: an initially homogeneous mixture can become heterogeneous if the cavity is shaken, and the opposite transition is also possible.

Within the zone of the spinodal decomposition the system becomes heterogeneous due to development of an infinitely small disturbance. Stepping outside of this zone, increasing the value of the average concentration, the size of the minimum disturbance capable of converting the system into the heterogeneous state ('the critical cluster' for the nucleation theory) grows. This minimum disturbance is of the maximum size for the values of the average concentration on the nucleation line. The size of such an disturbance is equal to the size of the smallest droplet that could remain in the container in equilibrium, termed 'the critical droplet' in the current work. The radius of this droplet $r_{c}$ is defined by figure 1c. A single droplet with the size smaller than $r_{c}$ may not remain in equilibrium in a closed container.

The obtained result is particularly important for the smaller enclosures, when dissolution of the solute additive may not occur as expected, or may occur at different, much slower, rate. Moreover, an experiment conducted with the use of the containers of different sizes would in general produce different phase diagrams for the same binary mixture. Normally, the phase diagram is reported as a property of the mixture. Our results show that the diagram can be also affected by the dimensions of the container.

It is interesting to relate the results obtained for the closed cavity to the states of the mixture in an unbounded domain. The size of the cavity, $L$, was used for the nondimensionalization of the governing equations, and it is then convenient to use the dimensional relations for the analysis of the asymptotic dependencies on $L$. For the analysis we use the asymptotic relations obtained for the smaller capillary numbers, that correspond to the case of larger cavities $\left(\mathrm{Ca} \rightarrow L^{-2}\right)$.

First, the critical curve that defines the stability of the 
homogeneous state in respect to finite-size perturbations is

$$
q=0.5-\left(\frac{\epsilon}{L^{2} \mu_{*}}\right)^{3 / 8} \rightarrow 0.5 .
$$

The similar asymptotic relations exist for the average concentrations in and outside of the droplet,

$$
\begin{gathered}
C_{1}=-0.5+1.2\left(\frac{\epsilon}{L^{2} \mu_{*}}\right)^{1 / 4} \rightarrow-0.5, \\
C_{2}=0.5+0.8\left(\frac{\epsilon}{L^{2} \mu_{*}}\right)^{3 / 8} \rightarrow 0.5 .
\end{gathered}
$$

The thickness of the interface and the surface tension coefficients do not depend on the size of the container,

$$
\delta_{*}=0.8\left(\frac{\epsilon}{\mu_{*}}\right)^{1 / 2}, \sigma_{*}=0.2 \rho_{*}\left(\mu_{*} \epsilon\right)^{1 / 2} .
$$

Here $\delta_{*}$ and $\sigma_{*}$ are the dimensional values of the interface thickness and surface tension coefficient, and $\rho_{*}$ is the typical density (e.g. density of solvent).

The most interesting observation is obtained for the size of the critical droplet,

$$
r_{c *}=0.6 L^{1 / 2}\left(\frac{\epsilon}{\mu_{*}}\right)^{1 / 4}=0.75\left(L \delta_{*}\right)^{1 / 2} .
$$

Here $r_{c *}$ is the dimensional size of the smallest droplet that may remain in equilibrium in a closed cavity.

As shown, if the container is very large then the boundary of the nucleation zone shifts to the value $1 / 2$. At the same time, the size of the critical droplet becomes very large, which means that the binary system may not be in an equilibrium heterogeneous state if the average concentration is near $1 / 2$, which is obvious. For the lower values of $q$ the nucleation would be possible. The minimum disturbance needed to convert the state of the system would depend on the average concentration, growing from zero at $q=1 / \sqrt{12}$ to the critical size $(21)$ at $q \approx 1 / 2$.

The phenomenological parameters of the phase-field approach, $\epsilon$ and $\mu_{*}$, cannot be directly measured. The estimations [24] show that $\epsilon \sim 10^{-13} \mathrm{~m}^{4} \cdot \mathrm{s}^{-2}$ and $\mu_{*} \sim$ $0.1 \mathrm{~J} \cdot \mathrm{kg}^{-1}$, which gives us $r_{c} \sim 10^{-3} \mathrm{~L}^{1 / 2}$. For a container with the size $L \sim 1 \mathrm{~cm}$, the radius of the critical nucleus is $r_{c} \sim 10^{-4} \mathrm{~m}$. The values of the interface thickness and the surface tension coefficients can be estimated as $\delta_{*} \sim 10^{-6} \mathrm{~m}$ and $\sigma_{*} \sim 10^{-5} \mathrm{~J} \cdot \mathrm{m}^{-2}$.

The financial support the Government of Perm region, Russia (Contract C-26-004-03) is gratefully acknowledged.

[1] P. He, S. J. Haswell, and P. D. I. Fletcher, Lab Chip 4, 38 (2004).
[2] R. Viskanta, M. A. Bianchi, J. K. Critser, and D. Gao, Cryobiology 34, 348 (1997).

[3] L. Granasy, Journal of Molecular Structure 485-486, 523 (1999).

[4] J. W. Gibbs, The Scientific Papers, Vol. I (Longmans Green and Company, New York, 39 Paternoster Row, London, 1928).

[5] J. W. Cahn and J. E. Hilliard, J. of Chem. Phys. 31, 688 (1959).

[6] R. C. Tolman, The Journal of Chemical Physics 17, 333 (1949).

[7] D. H. Rasmussen, J. Cryst. Growth 56, 45 (1982).

[8] J. Schmelzer, J. Chem. Soc., Faraday Trans. I 82, 1421 (1986).

[9] V. G. Baidakov and G. S. Boltachev, Phys. Rev. E 59, 469 (1999).

[10] J. W. P. Schmelzer, I. Gutzow, and J. J. Schmelzer, J. Colloid Interf. Sci. 178, 657 (1996).

[11] E. Santiso and A. Firoozabadi, Thermodynamics 52, 311 (2006).

[12] H. Gouin and M. Slemrod, Mechanica 30, 305 (1995).

[13] V. S. Borisevich, D. V. Lyubimov, and S. V. Shklyaev, in Hydrodynamics: Book of papers. Vol. 12 (Perm State University, 1999), pp. 62-78.

[14] M. Rao and B. J. Berne, J. Chem. Phys. 68, 1325 (1978).

[15] V. Talanquer and D. W. Oxtoby, J. Chem. Phys. 100, 5190 (1994).

[16] A. S. Abyzov and J. W. P. Schmelzer, J. Chem. Phys. 127, 114504 (2007).

[17] Z. Kozisek, K. Sato, S. Ueno, and P. Demo, J. Chem. Phys. 134, 094508 (2011).

[18] J. W. P. Schmelzer and A. S. Abyzov, J. Chem. Phys. 134, 054511 (2011).

[19] J. D. van der Waals and P. Kohnstamm, Lehrbuch der Thermodynamik (Maas and Van Suchtelen, Leipzig, 39 Paternoster Row, London, 1908).

[20] J. Lowengrub and L. Truskinovsky, Proc. R. Soc. London, Ser. A 454, 2617 (1998).

[21] A. Vorobev, Phys. Rev. E 82, 056312 (2010).

[22] L. D. Landau and E. M. Lifshitz, Statistical Physics, 3rd Edition, Part 1 (Pergamon Press, 1980).

[23] B. T. Nadiga and S. Zaleski, European Journal of Mechanics B/Fluids 15, 885 (1996).

[24] A. Vorobev and A. Boghi, Journal of Colloid and Interface Science 482, 193 (2016).

[25] F. Dell'Isola, H. Gouin, and E. Seppecher, C. R. Acad. Sci. Paris 320, 211 (1995).

[26] F. Dell'Isola, H. Gouin, and G. Rotoli, Eur. J. Mech. B/Fluids 15, 545 (1996).

[27] Another interesting point is that the matter is generally unstable when the diffusion coefficient, $\left(\partial \mu_{0} / \partial C\right)_{T}=$ $2 A+12 q^{2}$, is negative. This condition is in agreement with the obtained result of the phase-field theory except for the limit of very large capillary numbers. In the latter case, due to surface tension term, a mixture does remain homogeneous even if $\left(\partial \mu_{0} / \partial C\right)_{T}<0$, which may be important for a near-critical medium, as it permits the medium to remain homogeneous in a small container (with the size $L<p_{0} \sqrt{-\epsilon / 2 a}$, that increases as $\sim 1 / \sqrt{T_{c}-T}$ in the vicinity of the critical point) even when the diffusive coefficient is negative.

[28] These formulae are written in analogy with the formulae accepted in $[25,26]$. The radius of the droplet, though, 
was also calculated on the basis of the different approach: the level of concentration $C=0$ separates the phases, that may be used to identify the droplets from snapshots of the concentration fields. We found that, if the cap- illary number is sufficiently small, the difference in the results obtained on the bases of these two approaches is negligible. 